\title{
Osteotomy and iliac bone graft for the treatment of malunion caused by failed mandibular fracture reduction
}

\author{
Kun Hwang, \\ Sung Hwan Ma \\ Department of Plastic Surgery, Inha \\ University School of Medicine, Incheon, \\ Korea
}

\begin{abstract}
This report describes osteotomy and iliac bone graft for malunion caused by failed mandibular fracture reduction. A 27-year-old man was referred 3 months after a motor vehicle accident. At another hospital, two operations had been performed for symphyseal fracture using two resorbable plates. Malocclusion was noted, and panoramic radiography and computed tomography revealed a misaligned dental arch, with a 9.37-mm gap between the central and the lateral incisor of the left mandible. A wafer was made from the patient's dental model, and a maxillary arch bar was applied. Through a lower gingivolabial incision, osteotomy was performed between the malunited symphyseal fracture segments. Both segments were reduced to their original position using the wafer and fixed with titanium miniplates via intermaxillary fixation (IMF). The intersegmental gap was filled with cancellous bone from the iliac crest. The gingival defect was covered with a mucosal transposition flap from the gingivolabial sulcus. IMF and the wafer were maintained for 5 and 9 weeks, respectively. At postoperative week 13, the screws were removed from the mandible and satisfactory occlusion was noted. His mouth opening improved from 2.5 to 3 finger breadths $(40 \mathrm{~mm})$. This case demonstrates the need for sufficient IMF when using resorbable plates.
\end{abstract}

Keywords: Bone transplantation / Fractures, malunited / Mandible

\section{INTRODUCTION}

Various complications occur when a maxillofacial fracture is malunited or improperly resolved. Malocclusion is the most common complication, followed by facial deformity, temporo-

Correspondence: Kun Hwang

Department of Plastic Surgery, Inha University School of Medicine, 27 Inhang-ro, Jung-gu, Incheon 22332, Korea

E-mail: jokerhg@inha.ac.kr

This work was supported by a grant from National Research Foundation of Korea (NRF-2020R1I1A2054761).

We thank Hye Won Hu, BA, Division of Biomedical Art, Incheon Catholic University Graduate School, for her illustrations. We are also grateful to Hun Kim, Ph.D., Department of Plastic Surgery, Inha University School of Medicine, for editing the figures.

Received July 28, 2020 / Revised December 15, 2020 / Accepted December 20, 2020 mandibular joint disorder, and neurological symptoms. Nonetheless, very few papers have discussed the surgical treatment of malocclusion following mandibular fractures. This paper presents a case in which osteotomy and iliac bone grafting were performed to treat malunion caused by failed mandibular fracture reduction.

\section{CASE REPORT}

A 27-year-old man was referred to the authors' plastic surgery department. Three months previously, he had been hit by a motor vehicle while crossing a street and transferred to a regional trauma center. His initial Facial Injury Severity Scale (FISS) score was 2. A symphyseal fracture was noted, and two 
operations were performed using two resorbable plates at another hospital.

A clinical examination demonstrated malocclusion. His mouth opening was 2.5 finger breadths (Fig. 1A). Panoramic radiography and computed tomography scans revealed a misaligned dental arch of the mandible, with a $9.37-\mathrm{mm}$ gap between the left central and lateral incisors, where the right segment was displaced frontally (Fig. 2A). Before surgery, a wafer
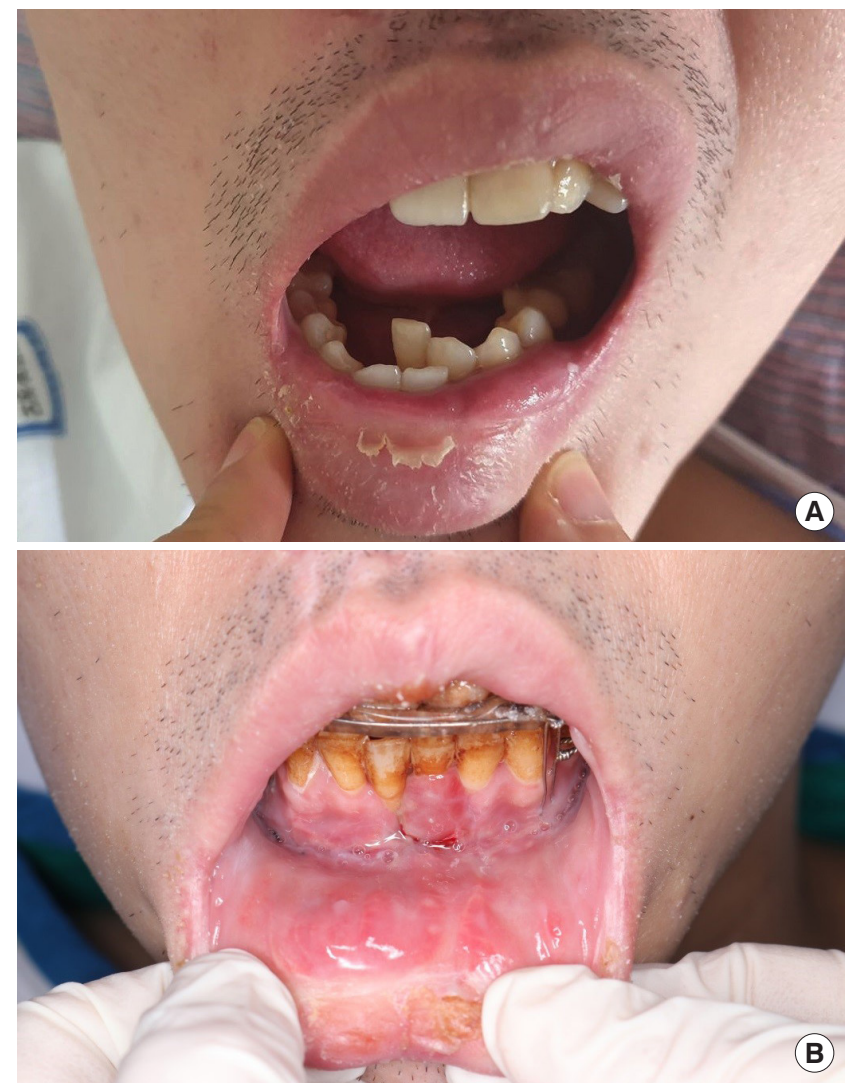

Fig. 1. Patient's photographs. (A) Preoperative. (B) Postoperative day 19. was made from the patient's dental model, and a maxillary arch bar was applied.

Under general anesthesia, a lower gingivolabial incision was made and osteotomy was performed between the malunited symphyseal fracture segments (Fig. 3A). Both segments were reduced to their original position using the wafer and fixed with titanium miniplates and intermaxillary fixation (IMF). The gap between the segments was filled with cancellous bone from the iliac crest (Fig. 3B). The gingival defect was covered with a mucosal transposition flap from the gingivolabial sulcus
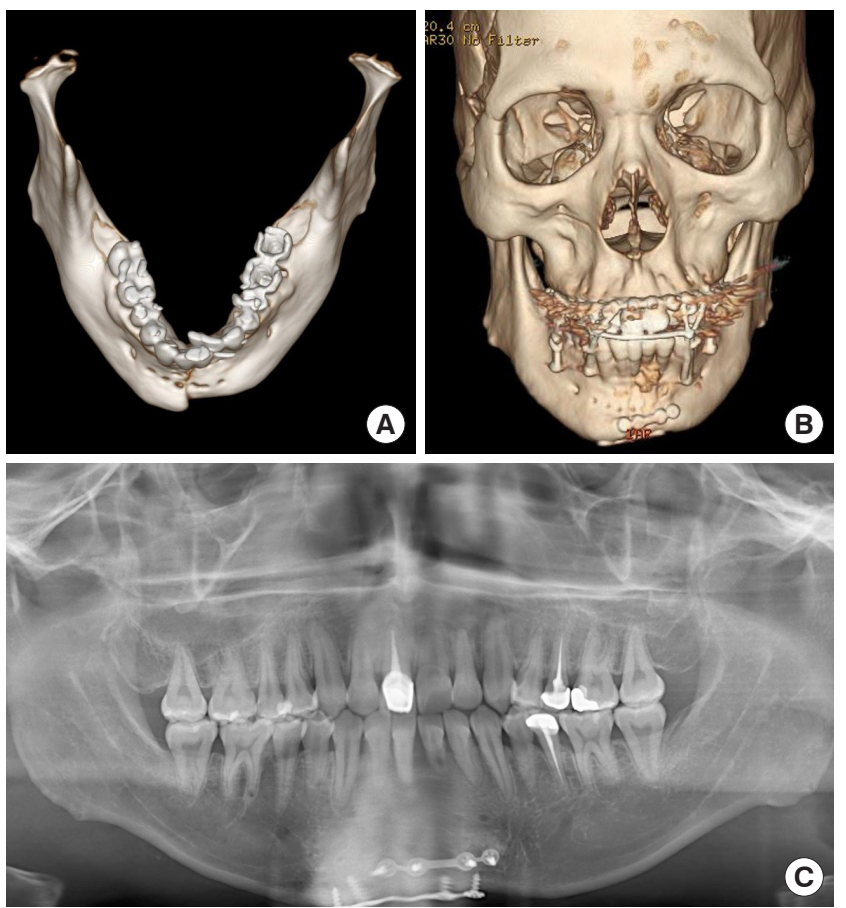

Fig. 2. Preoperative and postoperative radiologic images. (A) Three-dimensional preoperative computed tomography (CT). (B) CT on postoperative day 21. (C) Panoramic view on postoperative day 133.
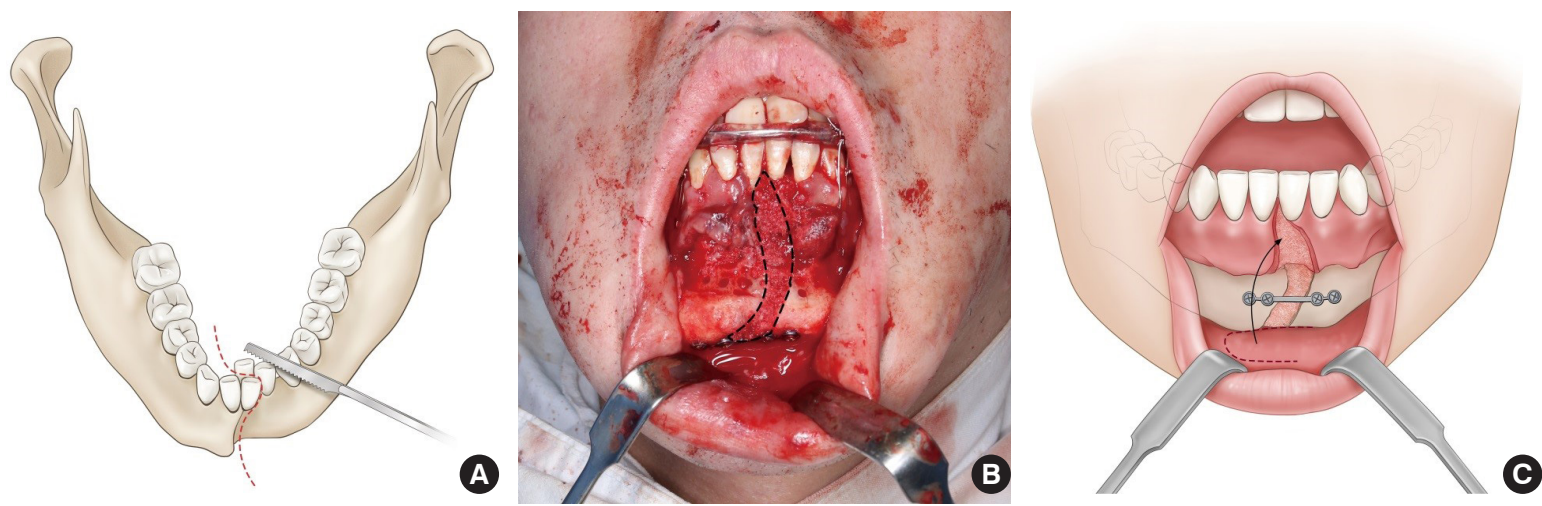

Fig. 3. Operative methods. (A) Osteotomy between malunited symphyseal fracture segments. (B) Both segments were reduced to their original position on the wafer and fixed with titanium miniplates. The gap was filled with cancellous bone (dotted line). (C) The gingival defect was covered with a mucosal transposition flap from the gingivolabial sulcus. 
(Fig. 3C).

The patient's hospital course was uneventful and he was discharged on postoperative day 25. His IMF was maintained for 5 weeks. After performing exercises, the maxillary arch bar was removed 7 weeks after the operation. The wafer was kept in place until the 9 th postoperative week. Thirteen weeks after the operation, the screws (SAS, skeletal anchorage system; Jeil Medical Co., Seoul, Korea) were removed from the mandible. Satisfactory occlusion was noted (Fig. 2B and C) with a mouth opening of 3 finger breadths (Fig. 1B).

\section{DISCUSSION}

Malunion is defined as bone union in an inaccurately reduced position. Malocclusion is the most common sign of malunion [1]. If malunion is found early, the fractured segments should be re-reduced and fixed, or the maxillomandibular fixation should be loosened so that occlusion can be restored [2]. In a case of failed miniplate fixation for an atrophic mandibular fracture, Santos used a load-bearing reconstruction plate combined with an autogenous bone graft from the iliac crest [3].

In the present case, involving a young adult, the trauma was more severe than was implied by the FISS score, as has been shown in recent research [4]. The failure in this case is thought to have been caused by fracture of the resorbable miniplates and early removal of the IMF. We mobilized the malunited fragments, placed them in their original position using a prefabricated wafer, and immobilized them with titanium miniplates. The bony defects were filled with cancellous bone graft. The IMF was maintained for 5 weeks and the wafer was kept in the lower jaw for 9 weeks. Based on this case, we suggest that when using resorbable plates for symphyseal fractures, sufficient IMF is needed.

The ilium is the most commonly used site for corticocancellous bone grafts. The clinical indications of grafts from the ilium are broad because the ilium is versatile and can provide large amounts of cortical and cancellous bone. The iliac crest is relatively easily accessible and has limited morbidity if the proper technique is used. However, several complications have been commonly reported, including chronic pain at the donor site, sensory changes, and gait abnormalities [5].

\section{NOTES}

\section{Conflict of interest}

No potential conflict of interest relevant to this article was reported.

\section{Ethical approval}

The study was approved by the Institutional Review Board of Inha University Hospital (2020-08-004-000) and performed in accordance with the principles of the Declaration of Helsinki. Written informed consent was obtained.

\section{Patient consent}

The patient provided written informed consent for the publication and the use of his images.

\section{ORCID}

Kun Hwang https://orcid.org/0000-0002-1994-2538

Sung Hwan Ma https://orcid.org/0000-0002-1956-9016

\section{REFERENCES}

1. Ellis E 3rd. Complications of rigid internal fixation for mandibular fractures. J Craniomaxillofac Trauma 1996;2:32-9.

2. Kim SY, Choi YH, Kim YK. Postoperative malocclusion after maxillofacial fracture management: a retrospective case study. Maxillofac Plast Reconstr Surg 2018;40:27.

3. Santos GS, de Assis Costa MD, de Oliveira Costa C, Souza FA, Junior IR, de Melo WM. Failure of miniplate osteosynthesis for the management of atrophic mandibular fracture. J Craniofac Surg 2013;24:e415-8.

4. Lee H, Kim KS, Choi JH, Hwang JH, Lee SY. Trauma severity and mandibular fracture patterns in a regional trauma center. Arch Craniofac Surg 2020;21:294-300.

5. Kurz LT, Garfin SR, Booth RE Jr. Harvesting autogenous iliac bone grafts: a review of complications and techniques. Spine (Phila Pa 1976) 1989;14:1324-31. 